Algebraic $\& \mathcal{G}$ Geometric Topology

Volume 2 (2002) 825-842

Published: 8 October 2002

ATG

\title{
3-manifold invariants and periodicity of homology spheres
}

\author{
PATRICK M. GiLmer \\ JoAnna Kania-BARTOSZYNSKA \\ JÓZEF H. PRZYTYCKI
}

\begin{abstract}
We show how the periodicity of a homology sphere is reflected in the Reshetikhin-Turaev-Witten invariants of the manifold. These yield a criterion for the periodicity of a homology sphere.
\end{abstract}

AMS Classification 57M60, 57M27; 57M25, 57R56, 17B37

Keywords 3-manifolds, links, group actions, quantum invariants

"... the angel of topology and the devil of abstract algebra fight for the soul of each individual mathematical domain"

Herman Weyl $[\mathrm{W}]$

\section{Introduction}

Since 1988, we have witnessed a fascinating development in the theory of 3 manifold invariants: from Witten's ideas to Ohtsuki's finite type invariants. In this paper we present one of the few nontrivial applications of this theory by showing how the periodicity of a 3-manifold is reflected in its quantum invariants.

In section 2 we recall some necessary conditions for periodicity of links in terms of the Jones polynomial and the Kauffman bracket. Section 3 contains Theorem 7 which states that periodic homology spheres can be presented as results of surgeries on periodic links. We also relate periodic homology spheres to free cyclic covers of homology $S^{1} \times S^{2}$ 's. In section 4 we recall the definition and some properties of the $S O(3)$-quantum invariants associated to a odd prime $p$. These are versions of the 3-manifold invariants first described by Witten [Wi], and shown to be well-defined by Reshetikhin-Turaev [R-T]. Section 5 contains Theorem 14 which describes how the existence of a $\mathbb{Z}_{p}$ action on a homology sphere is reflected in the Reshetikhin-Turaev-Witten invariants associated to 
$p$-th roots of unity. Related results for $\mathbb{Z}_{p}$ actions when the order of the root of unity is co-prime to $p$ are discussed in [G1] and [C].

In the last section we apply our criterion to the Brieskorn homology spheres, $\Sigma(2,|n|,|2 n-1|)$. In particular, we show that the Poincaré homology sphere is not $p$-periodic where $p$ is any prime between 7 and 61 (where we stopped the computer).

For the convenience of the reader, we include an appendix written by the first author with a direct proof of Theorem 13 . The original proof in [G1] was given in a more general context.

Research on this paper was partially supported by NSF-DMS-9626818, NSFDMS-9971905, NSF-DMS-0203486 and by USAF grant 1-443964-22502.

\section{Periodic Links}

In this section we recall criteria for periodicity of links in $S^{3}$ in terms of the Jones polynomial and the Kauffman bracket. These criteria motivated our main result, Theorem 14.

Let $n$ be an integer greater than 1 .

Definition 1 A link $L$ in $S^{3}$ is called $n$-periodic if there is a smooth faithful $\mathbb{Z}_{n}$-action on $S^{3}$, with a circle as the fixed point set, that maps $L$ onto itself, and where $L$ is disjoint from the fixed point set. Furthermore, if $L$ is an oriented link, one assumes that a generator of $\mathbb{Z}_{n}$ preserves the orientation of $L$ or changes it to the opposite one.

Given $n$-periodic link, we know by the positive solution of the Smith Conjecture [M-B], that the fixed point set $\gamma$ of the $\mathbb{Z}_{n}$ action is an unknotted circle and this action is conjugate to an orthogonal action on $S^{3}$. In other words, if we identify $S^{3}$ with $\mathbb{R}^{3} \cup \infty$, then the fixed point set can be assumed to be equal to the "vertical" axis together with $\infty$. Then the generator of $\mathbb{Z}_{n}$ is the rotation $\varphi(z, t)=\left(e^{2 \pi i / n} \cdot z, t\right)$, where the coordinates on $\mathbb{R}^{3}$ come from the product of the complex plane and the real line, $\mathbb{C} \times \mathbb{R}$. The $n$-periodic link may be represented by a $\varphi$-invariant diagram as in Figure 1.

Let $L_{+}, L_{-}, L_{0}$ denote three oriented links that are identical except in some 3 -ball as shown in Figure 2. The Jones polynomial [J] of oriented links, $V_{L}(t) \in$ $Z\left[t^{ \pm \frac{1}{2}}\right]$, is defined recursively as follows:

Algebraic $\mathcal{E} \mathcal{G}$ Geometric Topology, Volume 2 (2002) 


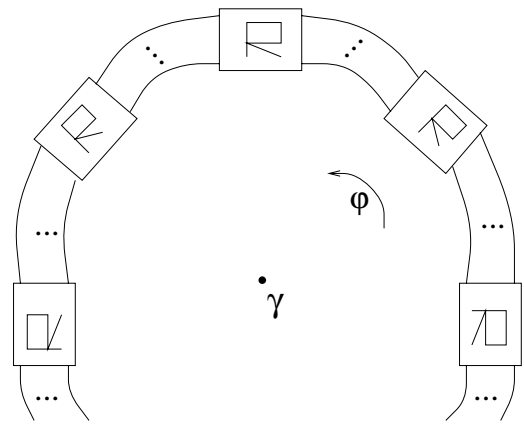

Figure 1: Periodic link diagram

(i) $V_{o}=1$,

(ii) $\frac{1}{t} V_{L_{+}}(t)-t V_{L_{-}}(t)=\left(\sqrt{t}-\frac{1}{\sqrt{t}}\right) V_{L_{0}}(t)$.

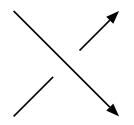

$\mathrm{L}_{+}$

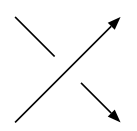

$\mathrm{L}$

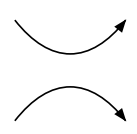

$\mathrm{L}_{0}$

Figure 2: Skein triple

The Kauffman bracket $[\mathrm{Ka}]$ is a function on the set of unoriented framed links in $\mathbb{R}^{3}$. It takes values in the ring $\mathbb{Z}\left[A^{ \pm 1}\right]$ and is uniquely determined by the rules:

(1)

$$
\langle\underbrace{\bigcirc \cdots \bigcirc}_{m}\rangle=\left(-A^{2}-A^{-2}\right)^{m-1} \text { and }
$$

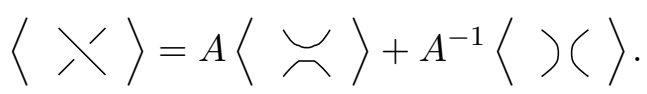

We assume, as usual, that the diagrams in condition (2) are identical in the parts not shown, and that the links are given the blackboard framing. The Kauffman bracket yields a simple definition of the Jones polynomial for oriented links. Let $L$ be an oriented link in $S^{3}$. For $L$ equipped with some framing the writhe $w(L)$ (or Tait number) of $L$ is the linking number of the oriented link $L$ and the push-off of $L$ given by the framing of $L$. We have

$$
V_{L}(t)=\left(-A^{3}\right)^{-w(L)}\langle L\rangle,
$$


where $A=t^{-\frac{1}{4}}$.

One of the notable applications of polynomial knot invariants is a criterion for periodicity discovered by Murasugi [M], Przytycki [P-1] and Traczyk [T-1], as given in the following theorem.

Theorem 2 Let $p$ be a prime number, and let $L$ be a $p$-periodic oriented link in $S^{3}$. Then

$$
V_{L}(t) \equiv V_{L}\left(t^{-1}\right) \quad \bmod \left(p, t^{p}-1\right)
$$

where $V_{L}(t)$ denotes Jones polynomial in variable $t$, and $\left(p, t^{p}-1\right)$ is the ideal in $\mathbb{Z}\left[t^{ \pm \frac{1}{2}}\right]$ generated by $p$ and $t^{p}-1$.

One obtains a similar criterion involving the Kauffman bracket.

Corollary 3 Let $L$ be a $p$-periodic oriented link where $p$ is prime. Assume $L$ has a framing which need not be periodic. We have that

$$
\langle L\rangle_{A} \equiv A^{6 w(L)}\langle L\rangle_{A^{-1}} \bmod \left(p, A^{4 p}-1\right),
$$

where we indicate by a subscript that we change the variable from $A$ to $A^{-1}$.

Recall that a framed link is an embedding of a disjoint collection of annuli into $S^{3}$. In diagrams we assume blackboard framing. We say that a framed link is $n$-periodic if it has an $n$-periodic diagram. In particular, an $n$-periodic framed knot must have writhe divisible by $n$.

Since $w(L) \equiv 0(\bmod p)$ for a $p$-periodic oriented framed link, we have

Corollary 4 If $p$ is prime, $L$ is a $p$-periodic (unoriented) framed link, and $A$ is a $2 p$-th root of unity, then

$$
\langle L\rangle \equiv \overline{\langle L\rangle} \bmod (p) .
$$

Here and below $\bar{z}$ denotes the complex conjugate of $z$.

This last result motivates Theorem 14 which gives a condition that has to be satisfied by the quantum invariant of a $p$-periodic pair $(M, L)$, where $M$ is a homology 3 -sphere, $L$ is a colored framed link in $M$, and $p$ is an odd prime. On the other hand, Corollary 4 follows from the proof of Theorem 14 by taking $M$ to be the 3 -sphere, and coloring $L$ with one. 


\section{Periodic Manifolds}

In this section we show that any periodic homology sphere can be presented as the result of surgery on a periodic link. We use this to observe that any periodic homology sphere can be obtained by doing equivariant surgery along a knot in a simple covering space of a homology $S^{1} \times S^{2}$.

Let $n$ be an integer greater than 1 . A $\mathbb{Z}_{n}$ action is called semi-free if it is free outside of the fixed point set.

Definition 5 An orientable 3 -manifold $M$ is $n$-periodic if it admits smooth semi-free action of the cyclic group $\mathbb{Z}_{n}$ with a circle as the fixed point set.

We need the following elementary lemma. First fix some notation. Suppose that $\mathbb{Z}_{n}$ acts on $M$ with the fixed-point set equal to a circle $\gamma$. Denote the quotients by $M_{*}=M / Z_{n}$, and $\gamma_{*}=\gamma$, and the projection map by $q: M \rightarrow M_{*}$.

Lemma 6 The map $q_{*}: H_{1}(M) \rightarrow H_{1}\left(M_{*}\right)$ is an epimorphism.

Proof Let $x_{0} \in \gamma$. Since $x_{0}$ is a fixed-point of the action, any loop based at $q\left(x_{0}\right)$ lifts to a loop based at $x_{0}$. Thus $q_{\#}: \pi_{1}\left(M, x_{0}\right) \rightarrow \pi_{1}\left(M_{*}, q\left(x_{0}\right)\right)$ is an epimorphism, and since $H_{1}$ is an abelianization of $\pi_{1}$, the map $q_{*}$ is also an epimorphism.

Theorem 7 Let $M$ be a homology sphere. If $\mathbb{Z}_{n}$ acts faithfully on $M$ with the fixed-point set equal to a circle then $M$ is $n$-periodic and there exists an $n$-periodic framed link $L \subset S^{3}$ such that $M$ is the result of surgery on $L$, and the linking number of each component of $L$ with the fixed point set for the action implied by the $n$-periodicity of $L$ is equal to zero.

Proof It follows from Smith theory $[\mathrm{Br}]$ that any faithful $\mathbb{Z}_{n}$ action on a homology sphere which has a circle as a fixed point set has to be semi-free. Thus $M$ is $n$-periodic.

The quotient manifold $M_{*}$ can be represented as a result of surgery on some framed link $L_{*}$ in $S^{3}$. We can assume that $L_{*}$ satisfies the following conditions:

(1) $\gamma_{*} \cap L_{*}=\emptyset$;

(2) $\operatorname{lk}\left(L_{*}^{i}, \gamma_{*}\right)=0$, for any component $L_{*}^{i}$ of $L_{*}$;

(3) $\gamma_{*}$ is unknotted in $S^{3}$.

Algebraic 83 Geometric Topology, Volume 2 (2002) 
$L_{*}$ satisfying the conditions 1-3 can be obtained as follows: Let $L_{*}^{\prime}$ be a framed link in $S^{3}$ such that $M_{*}$ is a result of surgery on $L_{*}^{\prime}$. Let $\hat{L}_{*}$ denote the co-core of the surgery. Co-core is a central line, $\{0\} \times S^{1}$, of the solid torus, $D^{2} \times S^{1}$, attached to $S^{3}-\operatorname{int}\left(V_{L_{*}^{\prime}}\right)$ in order to obtain $M_{*}$. As usual, $V_{L_{*}^{\prime}}$ denotes a tubular neighborhood of $L_{*}^{\prime}$ in $S^{3}$. In particular $\hat{L}_{*}$ is a framed link in $M_{*}$ such that $S^{3}$ is a result of surgery on $\hat{L}_{*}$. Since $\gamma_{*}$ is homologically trivial in $M_{*}$ it bounds a surface $F_{*}$. We can use isotopy to push $\hat{L}_{*}$ outside of $F_{*}$, therefore $F_{*}$ survives surgery. It has boundary $\gamma_{*}$ and is disjoint from $L_{*}$ (obtained from $L_{*}^{\prime}$ ) in $S^{3}$. Conditions (1) and (2) follow.

Now we are ready to unknot $\gamma_{*}$ using the Kirby calculus $([\mathrm{K}],[\mathrm{F}-\mathrm{R}])$. Choose some orientation on $\gamma_{*}$. We can add unlinked components with framing \pm 1 to $L_{*}$ around each crossing of $\gamma_{*}$, making sure that arrows on $\gamma_{*}$ run opposite ways (i.e. the linking number of $\gamma_{*}$ with the new component of $L_{*}$ is zero). Use the K-move to change the appropriate crossings (see Figure 3), and thus unknot $\gamma_{*}$. Thus we obtained condition (3) without compromising conditions (1) and (2). Consider the $n$-fold cyclic branched covering of $S^{3}$ by $S^{3}$ over

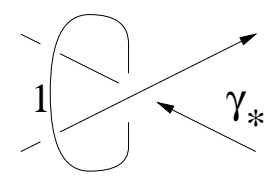

Figure 3: Unknotting $\gamma$

$\gamma_{*}$. Let $L$ denote the pre-image of $L_{*}$. Notice that $L$ is $n$-periodic. We claim that the result of performing surgery on $L$ is $\mathbb{Z}_{n}$-homeomorphic to $M$. The pre-image of each component of $L_{*}$ consists of $n$ components permuted by $\mathbb{Z}_{n}$ action, by condition (2). Therefore $\mathbb{Z}_{n}$ acts on the result of surgery on $S^{3}$ along $L,\left(S^{3}, L\right)$, with branched set $\gamma_{*}$ and quotient $\left(S^{3}, L_{*}\right)$. By Lemma 6 the manifold $M_{*}=\left(S^{3}, L_{*}\right)$ is a homology sphere. Therefore $H_{1}\left(M_{*}-\gamma_{*}\right)=\mathbb{Z}$, and thus $M_{*}-\gamma_{*}$ has unique $n$-fold cyclic covering. As a consequence, the branched covering of $\left(M_{*}, \gamma_{*}\right)$ is also unique. Thus $\left(S^{3}, L\right)$ is $\mathbb{Z}_{n}$-homeomorphic to $M$.

A $\mathbb{Z}_{n}$-covering space $X$ of a space $X_{*}$ is called simple if it is classified by a map $H_{1}\left(X_{*}\right) \rightarrow \mathbb{Z}_{n}$ which factors through an epimorphism $\chi$ to $\mathbb{Z}$ [G1, G2]. One can define a signature defect $[\mathrm{H}]$ of a simple covering $N \rightarrow N_{*}$ of closed orientable 3-manifolds as follows. Since $N \rightarrow N_{*}$ is a boundary of a simple $\mathbb{Z}_{n}$ covering space of 4 -manifolds $W \rightarrow W_{*}$, we may define $\operatorname{def}\left(N \rightarrow N_{*}\right)$ to be $\operatorname{def}\left(W \rightarrow W_{*}\right)=n \sigma\left(W_{*}\right)-\sigma(W)$. This does not depend on the choice of $W$. 
Remark 8 The third author has obtained a version of Theorem 7 for any $p$-periodic closed oriented 3-manifold $M$, where $p$ is prime. This appeared in [PS]. The third author and Sokolov also obtained a similar theorem for free $\mathbb{Z}_{p}$ actions [PS]. More recently Sakuma has shown that every orientation preserving finite cyclic action on a closed oriented 3-manifold $M$ has an equivariant framed link description [Sa].

Corollary 9 Suppose that $M$ is an $n$-periodic homology sphere. Then $M$ can be obtained by equivariant framed surgery along a knot in a simple covering of a homology $S^{1} \times S^{2}$. Moreover, the signature defect of such a covering is always even.

Proof Suppose that $\mathbb{Z}_{n}$ acts on $M$ with the fixed-point set equal to a circle $\gamma$. Let $L$ be a periodic framed link surgery description of $M$ given by Theorem 7 , and let $N$ be given by surgery on the framed link $L_{+}=L \cup \gamma$, where $\gamma$ has framing zero (i.e., $N$ is obtained by performing zero-framed surgery on $M$ along $\gamma$ ).

The free action on $M \backslash \gamma$ extends to a free $\mathbb{Z}_{n}$-action on $N$. Denote by $N_{*}$ the orbit space of this action. The manifold $N_{*}$ can be obtained by doing zero framed surgery on $M_{*}$ along $\gamma_{*}$. (We keep the notation from the proof of Theorem 7.) By Lemma 6, $M_{*}$ is a homology sphere, so $N_{*}$ is a homology $S^{1} \times S^{2}$. It follows that the quotient map $N \rightarrow N_{*}$ is a simple $\mathbb{Z}_{n}$-covering space. By [G1, Prop 4], def $\left(N \rightarrow N_{*}\right)$ is even.

Let $\delta$ be a meridian of $\gamma$ in $M$. The curve $\delta$ bounds a disk transverse to $\gamma$. We frame $\delta$ so its push-off bounds a transverse disk to $\gamma$ which misses the original transverse disk. If $\delta^{\prime}$ denotes the image of $\delta$ in $N$ under the surgery on $M$ along $\gamma$ then $M$ can be recovered from $N$ by doing surgery along $\delta^{\prime}$.

If $L$ is a framed link in $S^{3}$, the linking matrix of $L$ has off-diagonal entries given by the pairwise linking numbers of the components, and diagonal entries given by the linking numbers of the components with their push-offs according to the framing. Recall that $\sigma(L)$ denotes the signature of the linking matrix of $L$.

Remark 10 With the notation from the proof above, note that $L_{++}=L \cup \gamma \cup \delta$ is another framed link surgery description of $M$. We have that $\sigma(L)=\sigma\left(L_{+}\right)=$ $\sigma\left(L_{++}\right)$. 


\section{$4 \quad S O(3)$-Quantum Invariants at odd primes.}

Throughout the rest of this paper we assume $p$ is an odd prime. Let $M$ be a closed, connected, oriented 3-manifold, and $J$ a framed oriented $p$-colored link in $M$, i.e., a link whose components have been assigned nonnegative integers less than $p-1$. We begin this section by recalling the definition of the $S O(3)$ invariant $\mathcal{I}_{p}(M, J)$, following the description in $[\mathrm{M}-\mathrm{R}]$.

Suppose that $M$ is represented by a surgery on a framed link $L \subset S^{3}$, and that $J$ is disjoint from the co-cores of the surgery, so $J$ lies in $S^{3} \backslash L$. We use the renormalized Kauffman bracket

$$
[L]=-\left(A^{2}+A^{-2}\right)\langle L\rangle .
$$

Notice that $[\emptyset]=1$. Set the variable $A$ to be a primitive root of unity of order $2 p$. We denote by $k$ a root of unity such that

$$
k^{2}=A^{-6-p(p+1) / 2} .
$$

If $p \equiv-1(\bmod 4)$ then $\mathbb{Z}[A, k]=\mathbb{Z}[A]$. If $p \equiv 1(\bmod 4)$ then $\mathbb{Z}[A, k]=$ $\mathbb{Z}[A, i]$. Elements $e_{i}$ of the skein module of a solid torus are defined recursively by $e_{0}=1, e_{1}=z$ and

$$
e_{i+1}=z e_{i}-e_{i-1},
$$

where $z$ is represented by a longitude of the torus. The value of the Kauffman bracket of the skein element $e_{i}$, when the solid torus is embedded in $S^{3}$ in a standard way, is given by

$$
\Delta_{i}=(-1)^{i} \frac{A^{2 i+2}-A^{-2 i-2}}{A^{2}-A^{-2}} .
$$

By $\Omega_{p}$ we denote the following element of the Kauffman bracket skein module of a solid torus:

$$
\Omega_{p}=\sum_{i=0}^{\frac{p-3}{2}} \Delta_{i} e_{i}
$$

Let

$$
\eta=k \frac{1}{2 p} A^{3}\left(A^{2}-A^{-2}\right) A^{\frac{p(p-1)}{2}} \sum_{m=1}^{2 p}(-1)^{m} A^{m^{2}} .
$$

which satisfies

$$
\eta^{2}\left[\Omega_{p}\right]=1, \quad \text { i.e } \quad \eta^{2}=\frac{-\left(A^{2}-A^{-2}\right)^{2}}{p} .
$$

Algebraic $\mathcal{E} \mathcal{G}$ Geometric Topology, Volume 2 (2002) 
Recall that the invariant $\mathcal{I}_{p}(M, J)$ is given by:

$$
\mathcal{I}_{p}(M, J)=k^{-\sigma_{L}}\left[L\left(\eta \Omega_{p}\right) \sqcup J\right] .
$$

Here $\left[L\left(\eta \Omega_{p}\right) \sqcup J\right]$ denotes the bracket of the skein obtained by replacing each component of $L$ by $\eta \Omega_{p}$, and replacing each component of $J$ by the skein $e_{i}$, where $i$ is the color of the component. If one sets $A=e^{2 \pi i\left(1+p^{2}\right) / 4 p}$, and chooses $k$ so that $\eta>0$, the invariant $\mathcal{I}_{p}$ is equal to the quantum $S O(3)$-invariant $\tau_{p}^{S O(3)}[\mathrm{K}-\mathrm{M}]$.

Our criteria for periodicity of links require that we consider invariants modulo $p$. From the definition it is only apparent that $\mathcal{I}_{p}(M, J) \in \mathbb{Z}\left[A, k, \frac{1}{p}\right]$. Thus we will need the following result proved by Murakami $[\mathrm{Mu}]$ when $J$ is empty and generalized to colored links by Masbaum and Roberts [M-R].

Theorem 11 Let $J$ be a framed $p$-colored link in $M$, then $\mathcal{I}_{p}(M, J) \in \mathbb{Z}[A, k]$. If $p \equiv-1(\bmod 4)$ or $\beta_{1}(M)$ is even (in particular if $M$ is a homology sphere) then $\mathcal{I}_{p}(M, J) \in \mathbb{Z}[A]$.

In the proof of our criterion we also use the following integrality result for closed 3 -manifolds with non-zero first Betti number. It was proved by Murakami [Mu], and by Cochran and Melvin [C-M] for empty $J$. In full generality it follows from Gilmer's [G1, Corollary 4].

We adopt the notation:

$$
\langle\langle M, J\rangle\rangle_{p}=\eta \mathcal{I}_{p}(M, J) .
$$

This is suggested by TQFT notation (see the Appendix).

Theorem 12 Suppose that $M$ is connected and has first Betti number nonzero, and $J$ is a framed $p$-colored link in $M$, then $\langle\langle M, J\rangle\rangle_{p} \in \mathbb{Z}[A, k]$.

\section{$5 \quad S O(3)$-Quantum Invariants and $\mathbb{Z}_{p}$-group actions}

We will show in this section how the $p$-periodicity of $M$ is reflected in quantum $S O(3)$-invariant at $p$, assuming that $p$ is an odd prime. We will need the following theorem of Gilmer [G1]. For the convenience of the reader, a proof of this theorem is sketched in an appendix. Notice, that if $N$ is a simple $\mathbb{Z}_{p}$ covering space of $N_{*}$, then both $N$ and $N_{*}$ have first Betti number nonzero. Thus, by Theorem 12, it makes sense to consider $\langle\langle N, J\rangle\rangle_{p}$ modulo $p$. 
Theorem 13 If $(N, J)$ is a simple $\mathbb{Z}_{p}$ covering space of $\left(N_{*}, J_{*}\right)$, where $N_{*}$ is connected with odd first Betti number, and $p$ is an odd prime, then for some $n \in \mathbb{Z}$, we have:

$$
\langle\langle N, J\rangle\rangle_{p}=k^{\operatorname{def}\left(N \rightarrow N_{*}\right)} n \bmod (p) .
$$

We are ready to state and prove our main theorem. We remark that this theorem is uninteresting in the case $p=3$ as the conclusion always holds whether $M$ is 3 -periodic or not.

Theorem 14 Suppose that $M$ is a homology sphere, and $J$ is a $p$-colored framed link in $M$. If $(M, J)$ is $p$-periodic and $p$ is an odd prime then

$$
\mathcal{I}_{p}(M, J)=A^{2 j} \cdot \overline{\mathcal{I}_{p}(M, J)} \bmod (p)
$$

for some integer $j$.

Proof We are using the notation introduced in the proof of Corollary 9. By Remark $10, M$ is the result of surgery along $L_{++}$, so:

$$
\mathcal{I}_{p}(M, J)=k^{-\sigma\left(L_{++}\right)}\left[L_{++}\left(\eta \Omega_{p}\right) \sqcup J\right] .
$$

Expanding $\delta$ cabled with $\eta \Omega_{p}$, where $\delta_{i}$ denotes $\delta$ colored with $i$, we obtain:

$$
\mathcal{I}_{p}(M, J)=k^{-\sigma\left(L_{+}\right)} \eta \sum_{i=0}^{\frac{p-3}{2}} \Delta_{i}\left[L_{+}\left(\eta \Omega_{p}\right) \sqcup J \sqcup \delta_{i}\right],
$$

which is equal to

$$
\sum_{i=0}^{\frac{p-3}{2}} \Delta_{i}\left(\eta k^{-\sigma\left(L_{+}\right)}\left[L_{+}\left(\eta \Omega_{p}\right) \sqcup J \sqcup \delta_{i}\right]\right)
$$

or, by the definition of $\langle\langle\rangle\rangle$,

$$
\sum_{i=0}^{\frac{p-3}{2}} \Delta_{i}\left\langle\left\langle N, J \sqcup \delta_{i}\right\rangle\right\rangle .
$$

Theorem 13 implies that, modulo $p$, this is

$$
\sum_{i=0}^{\frac{p-3}{2}} \Delta_{i} k^{\operatorname{def}\left(N \rightarrow N_{*}\right)} n_{i}
$$

where $n_{i} \in \mathbb{Z}$. Since, by Corollary $9, \operatorname{def}\left(N \rightarrow N_{*}\right)$ is even,

$$
\mathcal{I}_{p}(M, J)=k^{2 s} \sum_{i=0}^{\frac{p-3}{2}} n_{i} \Delta_{i} \quad(\bmod p),
$$

Algebraic 83 Geometric Topology, Volume 2 (2002) 
where $s \in \mathbb{Z}$. Noting that $\Delta_{i}=\overline{\Delta_{i}}$, we have that

$$
\mathcal{I}_{p}(M, J)=k^{4 s} \overline{\mathcal{I}_{p}(M, J)} \quad(\bmod p) .
$$

The proof shows that phase factor is $k^{2} \operatorname{def}\left(N \rightarrow N_{*}\right)$. If $M$ is $S^{3}$, this defect is zero, and we obtain Corollary 4, as a consequence of the proof of Theorem 14 .

One expects similar results for quantum invariants associated to other Lie groups. These results should be related to Traczyk-Yokota periodicity criteria involving skein polynomials $[\mathrm{P}-2],[\mathrm{T}-2],[\mathrm{Y}]$. Corollary 5 in [G1] is a first step in this direction. In fact, subsequent to an earlier version of this paper, the results of this paper have been generalized to quantum invariants associated to simple complex Lie algebras by Chen and Le [C-L].

There should be a criterion for periodicity of other 3-manifolds than homology spheres in terms of their $S O(3)$-invariants. At present, we do not know how to prove it since no version of Theorem 13 has been obtained for non-simple $\mathbb{Z}_{p}$ covers.

\section{Examples}

We illustrate Theorem 14 by considering the Poincaré homology sphere $\Sigma[\mathrm{Po}]$. It is well known that $\Sigma$ is 5 -periodic since it is the 5 -fold branched cyclic cover of $S^{3}$ along the trefoil knot. The 5-periodicity is also apparent by presenting $\Sigma$ as a result of surgery on a 5 -periodic link $L$ pictured in Figure 4 (compare [Go], [Ro]) with blackboard framing. One can compute $\mathcal{I}_{5}(\Sigma)$ from its description as

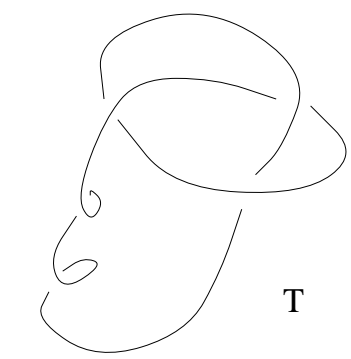

(a)

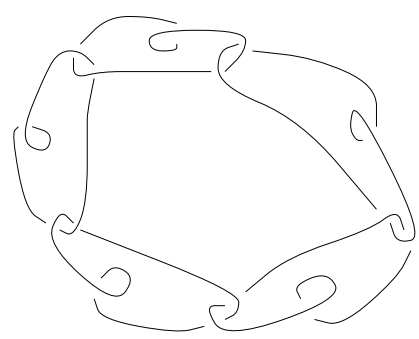

(b)

$\mathrm{L}$

Figure 4: Surgery descriptions of the Poincaré homology sphere

+1 surgery on the right handed trefoil [D] (knot $T$ in Figure 4):

$$
\mathcal{I}_{5}(\Sigma)=1-2 A+2 A^{2}-A^{3} .
$$

Algebraic $6 \mathcal{G}$ Geometric $\mathcal{T}$ opology, Volume 2 (2002) 
Thus

$$
\mathcal{I}_{5}(\Sigma)=A^{-2}\left(1-2 A^{-1}+2 A^{-2}-A^{-3}\right)=A^{-2} \overline{\mathcal{I}_{5}(\Sigma)}
$$

i.e. $j=-1$ in our criterion. It also follows from Theorem 14 that $\Sigma$ is not 7-periodic. We computed $\mathcal{I}_{7}(\Sigma)$ from the same surgery presentation.

$$
\mathcal{I}_{7}(\Sigma)=-2+A+2 A^{3}-A^{4}
$$

One can check that

$$
\mathcal{I}_{7}(\Sigma) \not \equiv A^{2 j} \overline{\mathcal{I}_{7}(\Sigma)} \quad(\bmod 7)
$$

for any $j$. Thus $\Sigma$ is not 7 -periodic.

More generally, consider the Brieskorn homology spheres $\Sigma(2,|n|,|2 n-1|)$, for $n$ odd. Let $K_{n}$ be a right handed $(2, n)$ torus knot if $n$ is positive, and a left handed $(2,-n)$ torus knot if $n$ is negative. Denote by $M_{n}$ the manifold obtained by +1 surgery along $K_{n}$. It was shown by Seifert in [S] that $M_{n}$ is a Seifert fibered homology sphere, with three exceptional fibers with multiplicities 2 , $|n|$, and $|2 n-1|$. Moreover, also according to [S], fixing $n$, there is only one homology sphere with such Seifert fibers (up to orientation). Thus $M_{n}$ is, up to orientation, the Brieskorn homology sphere $\Sigma(2,|n|,|2 n-1|)$. One can evaluate $\mathcal{I}_{p}\left(M_{n}\right)$ using the recoupling theory of [K-L, Chapter 9]. To obtain the value of the bracket of $K_{n}$ colored with $i$, use the first part of (9.15) to fuse the two strands, then apply (9.9) to untwist the resulting theta curve, and finally evaluate the resulting planar theta curve using (9.10). We obtain:

$$
\mathcal{I}_{p}\left(M_{n}\right)=\eta k^{-1} \sum_{i=0}^{\frac{p-3}{2}}\left((-A)^{i(i+2)(1-n)} \Delta_{i} \sum_{j=0}^{i} \Delta_{2 j}\left(\lambda_{2 j}^{i i}\right)^{-n}\right),
$$

where $[\mathrm{K}-\mathrm{L}, 9.9]$ :

$$
\lambda_{2 j}^{i i}=(-1)^{i-j} A^{i(i+2)-j(2 j+2)} .
$$

Lawrence derived the $\mathrm{SU}(2)$ invariants of this manifold in a related way [L].

We programmed Mathematica to evaluate $\mathcal{I}_{p}\left(M_{n}\right)$ symbolically and check whether the conclusion of Theorem 14 holds. Since $M_{n}$ is $p$-periodic for any odd prime $p$ dividing $n$ or $2 n-1$, we know the conclusion should hold in this case. We checked to see if the conclusion held for odd primes $5 \leq p \leq 19$, and for odd integers satisfying $3 \leq|n| \leq 19$. The condition held as expected whenever $p$ divided $n$ or $2 n-1$. This happened in 27 cases. It also held for the following list of ten other pairs of $(n, p):(9,5),(11,5),(13,7),(15,7),(19,5)$, $(-9,5),(-11,5),(-13,7),(-15,7),(-19,7)$. For the 71 other pairs in the above range, we can say $\Sigma(2,|n|,|2 n-1|)$ is not $p$-periodic. Thus we checked the conclusion for $6 \times 2 \times 9=108$ different pairs of prime $p$ and manifold $M$. 
The conclusion of Theorem 14 held in only 37 cases. In 27 of these cases the manifold is known to be $p$-periodic, as mentioned above.

In addition, we checked the criterion for the Poincaré homology sphere $\Sigma=M_{3}$ for larger values of $p$, and concluded that $\Sigma$ is not $p$-periodic if $p$ is any prime between 7 and 61 (where we stopped the computer).

Remark 15 Zhang has proven that the only periods for the Poincaré homology sphere are 2, 3, and 5, [Z]. More generally, we were informed by Boileau $[\mathrm{Bo}]$ that it follows from the orbifold theorem [Bo-Po] and the previous work of Meeks and Scott [M-S] and Seifert that if the Brieskorn homology sphere, $\Sigma(2,|n|,|2 n-1|)(|n|>2)$, is $Z_{p}$ periodic ( $p$ odd prime) then $p$ divides $n$ or $2 n-1$.

Remark 16 Chen and Le [C-L] checked that our periodicity criterion is also sufficient to prove that the only possible prime periods for the Poincaré homology sphere are 2,3 and 5 , and the only possible prime periods for $\Sigma(2,3,7)$ are 2,3 and 7 .

\section{References}

[BHMV1] C. Blanchet, N. Habegger, G. Masbaum, P. Vogel: Three manifold invariants derived from the Kauffman bracket, Topology 31 (1992), 685-699

[BHMV2] C. Blanchet, N. Habegger, G. Masbaum, P. Vogel: Topological quantum field theories derived from the Kauffman bracket, Topology 34 (1995), 883-927

[Bo] M. Boileau, Personal communication, Sept. 2001, March 2002.

[Bo-Po] M. Boileau, J. Porti: Geometrization of 3-orbifolds of cyclic type, Asterisque 272, (2001).

[Br] G. Bredon, Introduction to compact transformation groups, Academic Press, New York, 1972.

[C] N. Chbili : Les invariants $\theta_{p}$ des 3-varietes periodiques, preprint (2001).

[C-L] Q. Chen and T. Le: Quantum invariants of periodic links and periodic 3manifolds, preprint (2002).

[C-M] T. D Cochran, P. Melvin: Quantum cyclotomic orders of 3-manifolds, Topology, 40 (2001), no. 1, 95-125.

[D] M. Dehn: Über die Topologie des dreidimensionalen Raumes, Math. Annalen. 69, 137-168 (1910).

[F-R] R. Fenn, C. Rourke: On Kirby's calculus of links, Topology 18, 1-15 (1979).

[G1] P. M. Gilmer: Integrality for TQFTS, arXiv:math.QA/0105059

Algebraic 83 Geometric Topology, Volume 2 (2002) 
[G2] P. M. Gilmer: Quantum invariants of periodic three-manifolds, Proceedings of the Kirbyfest (Berkeley, CA, 1998), Geom. Topol. Monogr., 2, 157-175, (1999)

[Go] D. L. Goldsmith: Symmetric fibered links, in: Knots, groups and 3-manifolds ed.: L. P. Neuwirth, Princeton University Press, 1975.

$[\mathrm{H}] \quad$ F. Hirzebruch: The signature theorem: reminiscences and recreation, Prospects of Math, Ann. of Math Stud. 70, Princeton University Press, 3-31 (1971).

[Ja] N. Jacobson, Basic Algebra II, second edition, W.H. Freeman (1989)

[J] V. Jones: Hecke algebra representations of braid groups and link polynomials, Ann. of Math. 126, 335-388 (1987).

[Ka] L. Kauffman: On Knots, Annals of Mathematics Studies 115, Princeton University Press, 1987.

[K-L] L. Kauffman and S. Lins : Temperley-Lieb Recoupling Theory and Invariants of 3-manifolds, Annals of Mathematics Studies 134, Princeton University Press (1994).

[K] R. C. Kirby: A calculus for framed links in $S^{3}$, Invent. Math. 45, 35-56 (1978).

[K-M] R. C. Kirby, P. Melvin: The 3-manifold invariants of Witten and ReshetikhinTuraev for $s l(2, \mathbf{C})$. Invent. Math. 105, 473-545 (1991).

[L] R. J. Lawrence: Witten-Reshetikhin-Turaev invariants of 3-manifolds as holomorphic functions, Geometry and physics (Aarhus, 1995), Lecture Notes in Pure and Appl. Math., 184, 363-377, Dekker, New York, (1997).

[M-R] G. Masbaum, J. Roberts: A simple proof of integrality of quantum invariants at prime roots of unity, Math. Proc. Cambridge Philos. Soc., 121, 443-454 (1997).

[M-S] W. M. Meeks III, P. Scott,: Finite group actions on 3-manifolds, Invent. Math. 86(2), 287-346 (1986).

[M-B] J. W. Morgan, H. Bass, eds: The Smith conjecture, Pure Appl. Math. 112, New York Academic Press, 1984.

[Mu] H. Murakami: Quantum $S O(3)$-invariants dominate the $S U(2)$-invariant of Casson and Walker, Math. Proc. Cambridge Philos. Soc., 117, 237-249 (1995).

[M] K. Murasugi: Jones polynomials of periodic links, Pacific J. Math. 131, 319-329 (1988).

[Po] H. Poincaré: Cinquième Complément à l'Analysis Situs, Rend. Circ. Mat. Palermo 18, 277-308 (1904).

[P-1] J. H. Przytycki: On Murasugi's and Traczyk's criteria for periodic links, Math. Ann. 283, 465-478 (1989).

[P-2] J. H. Przytycki: An elementary proof of the Traczyk-Yokota criteria for periodic knots, Proc. Amer. Math. Soc. 123, 1607-1611 (1995).

[PS] J. H. Przytycki, M. Sokolov : Surgeries on periodic links and homology of periodic 3-manifolds. Math. Proc. Cambridge Philos. Soc. 131 , 295-307 (2001). 
[R-T] N. Yu. Reshetikhin, V. G. Turaev: Invariants of 3-manifolds via Link Polynomials and Quantum Groups, Invent. Math. 103, 547-597 (1991).

[Ro] D. Rolfsen: Knots and Links, Publish or Perish, (1976).

[Sa] M. Sakuma: Surgery description of orientation-preserving periodic maps on compact orientable 3-manifolds, Rend. Istit. Mat. Univ. Trieste XXXII, 1-11 (2000).

[S] H. Seifert : Topologie dreidimensional gefaserte Raume, Acta Math 60, 147-238 (1932).

[T-1] P. Traczyk: $10_{101}$ has no period 7: a criterion for periodicity of links, Proc. Amer. Math. Soc. 180, 845-846 (1990).

[T-2] P. Traczyk: Periodic links and the skein polynomial, Invent. Math. 106, 73-84 (1991).

[W] Herman Weyl: Invariants, Duke Mathematical Journal 5, 489-502 (1939).

[Wi] E. Witten: Quantum field theory and the Jones polynomial, Comm. Math. Phys. 121, 351-399 (1989).

[Y] Y. Yokota: The skein polynomial of periodic links, Math. Ann. 291, 281-291 (1991).

[Z] X. Zhang: On property I for knots in $S^{3}$, Trans. AMS 339, 643-657 (1993).

Department of Mathematics, Louisiana State University

Baton Rouge, LA 70803, USA

Department of Mathematics, Boise State University

Boise, ID 83725, USA

Department of Mathematics, George Washington University

Washington, D.C.20052, USA

Email: gilmer@math.lsu.edu, kania@math.boisestate.edu, przytyck@research.circ.gwu.edu

Received: 19 November 2001 Revised: 3 September 2002 


\title{
Appendix: Quantum invariants of simple free $\mathbb{Z}_{p}$ covers
}

\author{
PATRICK M. GILMER
}

As background for the proof of Theorem 13 we now describe some features of the topological quantum field theory defined in [BHMV2], and the relation of the functor $\left(V_{p}, Z_{p}\right)$ to $\mathcal{I}_{p}(M, J)$, and $\langle\langle M, J\rangle\rangle_{p}$.

The notion of a $p_{1}$-structure provides one solution to the problem of finding extra structures for surfaces and 3-manifolds so that one can remove the "framing anomalie" in the Reshetikhin-Turaev-Witten TQFTs. See [BHMV2] for the definition of $p_{1}$ structures.

For a closed 3-manifold $M$ with $p_{1}$-structure $\alpha$, there is an associated integer $\sigma(\alpha)$ which specifies the $p_{1}$-structure up to homotopy.

Let $\kappa$ be a cube root of $k$. Let $R_{p}$ be the $\operatorname{ring} \mathbb{Z}\left[A, \kappa, \frac{1}{p}\right]$. The functor $V_{p}$ assigns a free finitely generated module over $R_{p}$ to a surface $\Sigma$ equipped with a $p_{1}$-structure and some even-colored framed points. This module is denoted $V_{p}(\Sigma)$. Let $N$ be a 3 -dimensional cobordism with a framed even-colored link $\mathrm{J}$ and $p_{1}$-structure, whose boundary is identified with $-\Sigma_{1} \sqcup \Sigma_{2}$. Link $J$ must meet the boundary in the colored framed points. The functor $Z_{p}$ assigns to $N$ a module homomorphism $Z_{p}(N)$ : $V_{p}\left(\Sigma_{1}\right) \rightarrow V_{p}\left(\Sigma_{2}\right)$.

A closed 3-manifold $M$ with $p_{1}$-structure and an even-colored framed link $J$ can be considered as a cobordism from the empty set to the empty set. As $V_{p}(\emptyset)=R_{p}$, $Z_{p}(M)$ is multiplication by some scalar in $R_{p}$. This scalar is denoted $\langle M\rangle_{p}$. The invariant $\mathcal{I}_{p}(M, J)$ is a rescaled version of the invariant $\langle M\rangle_{p}$ with the dependence on the $p_{1}$-structure removed. One has:

$$
\langle M\rangle_{p}=\kappa^{\sigma(\alpha)} \eta \mathcal{I}_{p}(M, J),
$$

and

$$
\langle\langle M, J\rangle\rangle_{p}=\kappa^{-\sigma(\alpha)}\langle M\rangle_{p}
$$

Thus $\langle\langle M, J\rangle\rangle_{p}$ is $\langle\tilde{M}, J\rangle_{p}$, where $\tilde{M}$ is $M$ with a reassigned $p_{1}$-structure whose $\sigma$ invariant is zero. Note that when we use the quantum invariant notations: $\mathcal{I}_{p}$ and $\langle\langle\rangle\rangle_{p}$, we explicitly include $J$. However when using the TQFT notations: $Z_{p}$ and \langle\rangle$_{p}$, the symbols for the cobordisms $M$ and $N$ etc., implicitly include the data of $J$ and a $p_{1}$ structure.

If $\sigma(\alpha)=3\left(\beta_{0}(M)+\beta_{1}(M)\right)(\bmod 6)$, one has [BHMV2, 2$]\left[\right.$ BHMV1] that $\langle M, J\rangle_{p} \in$ $\mathbb{Z}\left[A, \frac{1}{p}\right]$.

Suppose that $E$ is a cobordism from $\Sigma$ to itself. Then one may form $C(E)$, the closure of $E$, by identifying the two copies of $\Sigma$ which make up the boundary. We have the important trace property:

$$
\langle C(E)\rangle_{p}=\operatorname{Trace}\left(Z_{p}(E)\right)
$$

Algebraic 63 Geometric Topology, Volume 2 (2002) 
If $H$ is a connected cobordism from $\emptyset$ to $\Sigma$, let $[\mathrm{H}]$ denote the image of $1 \in V_{p}(\emptyset)$ in $V_{p}(\Sigma)$ under $Z_{p}(H)$. The module $V_{p}(\Sigma)$ is finitely generated by such elements. Moreover there is a well-defined non-degenerate Hermitian form $\langle,\rangle_{\Sigma}$ on $V_{p}(\Sigma)$ given by

$$
\left\langle\left[H_{1}\right],\left[H_{2}\right]\right\rangle_{\Sigma}=\left\langle H_{1} \cup_{\Sigma}-H_{2}\right\rangle_{p} .
$$

This completes the summary of the properties of $\left(V_{p}, Z_{p}\right)$ that we need for the proof of Theorem 13.

Proof If $N_{*}$ and $N$ contain some link components with odd colors, trade them for

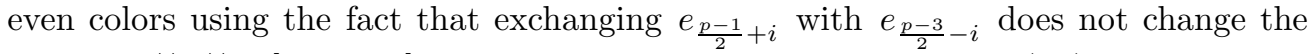
value of $\langle\langle N\rangle\rangle_{p}$ [BHMV1]. Give $N_{*}$ a $p_{1}$-structure $\alpha_{*}$, with $\sigma\left(\alpha_{*}\right)=0$, so that $\left\langle N_{*}\right\rangle_{p} \in \mathbb{Z}\left[A, \frac{1}{p}\right]$. By Theorem $12\left\langle\left\langle N_{*}, J_{*}\right\rangle\right\rangle_{p} \in \mathbb{Z}[A, k]$. Thus $\left\langle N_{*}\right\rangle_{p} \in \mathbb{Z}[A, k]$. Therefore $\left\langle N_{*}\right\rangle_{p} \in \mathbb{Z}[A, k] \cap \mathbb{Z}\left[A, \frac{1}{p}\right]=\mathbb{Z}[A]$.

$N$ acquires an induced $p_{1}$-structure $\alpha$. By [G2, Lemma 4], we have that $3 \operatorname{def}(N \rightarrow$ $\left.N_{*}\right)=-\sigma(\alpha)$. Since $k=\kappa^{3}$, we have that $\langle\langle N, J\rangle\rangle=k^{\operatorname{def}\left(N \rightarrow N_{*}\right)}\langle N\rangle_{p}$.

Since $N \rightarrow N_{*}$ is simple, it can be defined using reduction modulo $p$ of some epimorphism $\chi: H_{1}\left(N_{*}\right) \rightarrow Z$. Let $\Sigma$ be a connected surface in $N_{*}$ which is Poincare dual to the class of $\chi$ in $H^{1}\left(N_{*}\right)$. Let $E$ denote $N_{*}$ slit along $\Sigma$. The manifold $E$ is connected and we may regard $E$ as a cobordism from $\Sigma$ to $\Sigma$. We denote the composition in the cobordism category of $E$ with itself $n$ times by $E^{n}$. We have that $N_{*}=C(E)$, and $N$ is diffeomorphic to $C\left(E^{p}\right)$. Thus $\left\langle N_{*}\right\rangle_{p}=\operatorname{Trace}\left(Z_{p}(E)\right)$, and $\langle N\rangle_{p}=\operatorname{Trace}\left(\left(Z_{p}(E)\right)^{p}\right)$,

We will show below that there is a matrix $\mathcal{M}$ whose coefficients are algebraic integers such that $\operatorname{Trace}(\mathcal{M})=\operatorname{Trace}\left(Z_{p}(E)\right)$, and $\operatorname{Trace}\left(\mathcal{M}^{p}\right)=\operatorname{Trace}\left(Z_{p}(E)^{p}\right)$. Assuming the existence of $\mathcal{M}$, we now complete the proof.

We have that $\operatorname{Trace}(\mathcal{M})=\sum_{i} \lambda_{i}$, where $\left\{\lambda_{i}\right\}$ denotes the list of eigenvalues of $\mathcal{M}$, with multiplicities. Each $\lambda_{i}$ is an algebraic integer as it satisfies the characteristic polynomial of $\mathcal{M}$, whose coefficients are algebraic integers. Moreover $\operatorname{Trace}\left(\mathcal{M}^{p}\right)=$ $\sum_{i} \lambda_{i}^{p}$. Moreover $\sum_{i} \lambda_{i}^{p}-\left(\sum_{i} \lambda_{i}\right)^{p}$ is $p$ times an algebraic integer, since $\left(\begin{array}{l}p \\ j\end{array}\right)=0$ $(\bmod p)$ for $0<j<p$.

So $\langle N\rangle_{p}-\left(\left\langle N_{*}\right\rangle_{p}\right)^{p}$ is $p$ times an algebraic integer. As $\left\langle N_{*}\right\rangle_{p} \in \mathbb{Z}[A]$, we have that $\left(\left\langle N_{*}\right\rangle_{p}\right)^{p}$ is congruent modulo $p$ to an ordinary integer, say $n$. Thus $\langle N\rangle_{p}-n$ is $p$ times an algebraic integer. It follows that $\langle\langle N, J\rangle\rangle-k^{\text {def }\left(N \rightarrow N_{*}\right)} n$ is $p$ times an algebraic integer. This is the conclusion of the theorem.

Thus we only need to find $\mathcal{M}$. Let $S_{p}(\Sigma)$ denote the $\mathbb{Z}[A, \kappa]$ submodule of $V_{p}(\Sigma)$ generated by all elements of the form $[H]$ where $H$ is a connected cobordism from $\emptyset$ to $\Sigma$. As this set spans $V_{p}(\Sigma)$ over $R_{p}$, we have that $S_{p}(\Sigma) \otimes_{\mathbb{Z}[A, \kappa]} R_{p}=V_{p}(\Sigma)$.

We claim that $S_{p}(\Sigma)$ is a finitely generated projective $\mathbb{Z}[A, \kappa]$-module. To see this pick a finite set of generators: $\left\{\left[H_{i}\right]\right\}_{1 \leq i \leq m}$ for $V_{p}(\Sigma)$ where $H_{i}: \emptyset \rightarrow \Sigma$ are connected. We have an injective map

$$
S_{p}(\Sigma) \rightarrow \mathbb{Z}[A, \kappa]^{m}
$$


given by

$$
[H] \mapsto \frac{1}{\eta}\left(\left\langle[H],\left[H_{1}\right]\right\rangle_{\Sigma}, \cdots,\left\langle[H],\left[H_{m}\right]\right\rangle_{\Sigma}\right)
$$

The reason that this map takes values in $\mathbb{Z}[A, \kappa]^{m}$ is Theorem 11 . As $\mathbb{Z}[A, \kappa]$ is a Dedekind domain, a submodule of $\mathbb{Z}[A, \kappa]^{m}$ is necessarily finitely generated and projective [Ja, Proposition 10.12].

The $\mathbb{Z}[A, \kappa]$ submodule $S_{p}(\Sigma)$ of $V_{p}(\Sigma)$ is preserved by $Z_{p}(E): V_{p}(\Sigma) \rightarrow V_{p}(\Sigma)$. This is seen as follows. Given a connected $H: \emptyset \rightarrow \Sigma, Z_{p}(E)[H]=\left[H \cup_{\Sigma} E\right]$, and $H \cup_{\Sigma} E$ is connected. We denote the induced map by $\mathcal{Z}: S_{p}(\Sigma) \rightarrow S_{p}(\Sigma)$.

There is an ideal $\mathcal{J}$ in $\mathbb{Z}[A, \kappa]$ such that $S_{p}(\Sigma) \oplus \mathcal{J} \approx \mathbb{Z}[A, \kappa]^{l}$, where $l=\operatorname{dim} V_{p}(\Sigma)+1$ [Ja, Theorem 10.14]. So, denoting by $0_{\mathcal{J}}$ the zero map from $\mathcal{J}$ to itself,

$$
\operatorname{Trace}\left(Z_{p}(E)\right)=\operatorname{Trace}\left(\mathcal{Z} \oplus 0_{\mathcal{J}}\right)=\operatorname{Trace}(\mathcal{M}),
$$

where $\mathcal{M}$ is a matrix which represents the endomorphism $\mathcal{Z} \oplus 0_{\mathcal{J}}$ of free $\mathbb{Z}[A, \kappa]$ modules. Similarly

$$
\operatorname{Trace}\left(Z_{p}\left(E^{p}\right)\right)=\operatorname{Trace}\left(\mathcal{Z}^{p} \oplus 0_{\mathcal{J}}\right)=\operatorname{Trace}\left(\mathcal{M}^{p}\right) .
$$

